\title{
Diet quality of adolescents in rural Sri Lanka based on the Diet Quality Index-International: findings from the 'Integrating Nutrition Promotion and Rural Development' project
}

\author{
Julianne Williams ${ }^{1, *}$, Nick Townsend ${ }^{1}$, Mike Rayner $^{1}$, Ranil Jayawardena ${ }^{2}$, \\ Prasad Katulanda ${ }^{3}$, Seenithamby Manoharan ${ }^{4}$ and Kremlin Wickramasinghe ${ }^{1}$ \\ ${ }^{1}$ Centre on Population Based Approaches for Non-Communicable Disease Prevention, Nuffield Department of \\ Population Health, University of Oxford, Old Road Campus, Oxford OX3 7NX, UK: ${ }^{2}$ Department of Physiology, \\ Faculty of Medicine, University of Colombo, Colombo, Sri Lanka: ${ }^{3}$ Department of Clinical Medicine, Faculty of \\ Medicine, University of Colombo, Colombo, Sri Lanka: ${ }^{4}$ The World Bank, Colombo, Sri Lanka
}

Submitted 25 October 2017: Final revision received 30 November 2018: Accepted 3 January 2019: First published online 1 April 2019

\begin{abstract}
Objective: The current paper describes methods of evaluating dietary habits of Sri Lankan adolescents based on the Diet Quality Index-International (DQI-I), which has been used in multiple international studies to describe dietary variety, moderation, adequacy and balance. The paper describes the method for calculating DQI-I scores and examines associations between DQI-I scores and dietary intake, and between DQI-I scores and sociodemographic factors.

Design: The study followed a three-stage cluster randomised sampling method. Dietary intake was collected using a validated FFQ. Estimated micronutrient intakes and number of servings consumed were described according to DQI-I quartiles. DQI-I scores were tabulated according to sociodemographic characteristics. Multilevel modelling was used to examine associations between sociodemographic characteristics and DQI-I scores.

Setting: Secondary schools in rural Sri Lanka.

Participants: Adolescents ( $n$ 1300) aged 12-18 years attending secondary school in rural Sri Lanka.

Results: DQI-I scores increased with consumption of fat (\% energy), cholesterol $(\mathrm{mg} / \mathrm{d})$, energy $(\mathrm{kJ} / \mathrm{d})$, protein (\% energy), $\mathrm{Na}(\mathrm{mg})$, dietary fibre $(\mathrm{g}), \mathrm{Fe}(\mathrm{mg})$ and $\mathrm{Ca}$ (mg), but decreased according to percentage of energy coming from carbohydrates. DQI-I scores were significantly lower among females and students with lower levels of maternal education.

Conclusions: Policies are needed to increase the availability and affordability of nutrient-rich foods such as fruits, vegetables and high-protein foods, particularly to students from lower socio-economic backgrounds. Significant differences in diet quality according to sex, socio-economic status and district suggest there is potential for targeted interventions that aim to increase access to affordable, nutrient-rich foods among these groups.
\end{abstract}

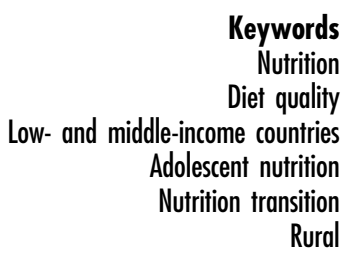

Nutrition ensures good health throughout the entire life course, but it is particularly important during adolescence, when individuals have increased nutrient needs to meet the demands of physical and cognitive growth and development. With adolescents, particular nutritional concerns include higher than recommended amounts of saturated fat and $\mathrm{Na}$, inadequate amounts of fruits, vegetables and fibre, and low intakes of $\mathrm{Ca}$ and Fe-rich foods, which are especially important for adolescent girls ${ }^{(1)}$.

Sri Lanka, like many low-to middle-income countries, is experiencing a rapid economic, demographic and epidemiological transition ${ }^{(2,3)}$ and a double burden of under- and overnutrition ${ }^{(4,5)}$. Within the population there is a high prevalence of metabolic syndrome ${ }^{(6)}$, diabetes ${ }^{(7)}$ and undernutrition ${ }^{(8,9)}$.

Consuming a high-quality diet is a challenge globally, but it is particularly difficult for individuals living in environments where nutrient-dense foods, such as those from animal sources or fresh fruits or vegetables, are unavailable or unaffordable ${ }^{(10)}$. According to a 2013 study, only $3.5 \%$ of adults in Sri Lanka consumed the recommended five daily portions of fruits and vegetables, over a 
third of the population consumed no daily dairy products and nearly $70 \%$ consumed over fourteen portions of starch daily $^{(11)}$.

One of the challenges that researchers working in lowto middle-income country contexts face relates to quantifying diet quality. In many studies, proxy measures such as the 'number of daily fruits and vegetables' or 'number of sugary drinks' are used to characterise diets. However, in certain instances, such as modelling exercises, it may be useful to have a quantitative estimate that characterises multiple aspects of dietary quality. The use of composite indices to summarise overall diet quality is becoming increasingly popular in nutrition and health research, with a marked increase in the number of studies using diet quality indices tailored to specific purposes and populations $^{(12)}$. Compared with single nutrient measures, composite measures provide a more comprehensive means of assessing variability and overall diet quality. Composite measures have been found to be associated with dietrelated biomarkers ${ }^{(13)}$ and may be more strongly associated with health outcomes than a single nutrient ${ }^{(14,15)}$. By providing a predefined summary measure of overall diet quality, diet quality indices can be used to monitor overall dietary changes based on a wide selection of foods and nutrients that are considered important for health $^{(12,16)}$.

At the same time, there is some controversy surrounding the utility of diet quality indices. Waijers et al. reviewed the literature on twenty predefined indices of overall diet quality and concluded that many correlations between the dietary components are not adequately addressed ${ }^{(17)}$. A second review by Arvaniti et al. also reviewed dietary indices and concluded that the majority of these indices fail to recognise the various interrelationships between their components ${ }^{(18)}$.

Another issue related to diet quality indices is the fact that there are many options; a review by Marshall et al. ${ }^{(19)}$ identified 119 studies using eighty different diet quality indices to assess diet among children and adolescents. For the present study, we decided to use the Diet Quality Index-International (DQI-I). The advantage of this index is that it considers issues related to both undernutrition (e.g. variety and adequacy) and overnutrition (moderation and balance), which is important when working in environments experiencing a nutrition transition. Many of the other dominant diet quality indices were not appropriate due to their focus on populations living in highincome countries where the primary health concern relates to overnutrition ${ }^{(19)}$.

The purpose of the current work was to describe the diet quality of adolescents in rural Sri Lanka using the DQI-I. Additionally, it aimed to examine how DQI-I scores related to dietary consumption in terms of types of foods consumed, macronutrient composition and micronutrient intakes. Finally, using multilevel analysis to account for the clustered sampling methods, it aimed to examine associations between sociodemographic characteristics and diet quality. We hypothesised that DQI-I scores would be positively associated with nutrient intakes and that scores would vary according to sociodemographic characteristics such as maternal education.

\section{Methods}

\section{Data collection}

Data were collected as part of the 'Integrating Nutrition Promotion and Rural Development' project, a quasiexperimental study funded by the World Bank which aimed to evaluate the feasibility of promoting healthy diets in rural Sri Lanka through a range of multisectoral activities. Data were collected from three districts: Ampara, Moneregala and Kurunegala.

Schools and students were sampled from the three districts using three-stage cluster sampling methods based on district, school type and whether or not the school was involved with the 'Integrating Nutrition Promotion and Rural Development' project. These methods resulted in a sample of fifty schools. Students were selected from schools using probability proportional to size, to ensure that every student had an equal chance of being selected. Students between the ages of 12 and 18 years attending secondary school were eligible to participate. According to WHO recommendations, study participants aged 12 years or above are eligible to give informed consent ${ }^{(20)}$. We excluded students under the age of 12 years, those diagnosed with long-term medical conditions and those who were attending private or international schools.

A week before the data collection, each participating school sent students' parents a letter providing detailed information about the project and instructions for opting out of the survey. On the day of the survey, students who had not opted out of the project were invited to participate. Those who agreed to participate provided written informed consent. Even after providing consent, participants were notified that at any point after giving written informed consent, they were eligible to opt out of the study. Ethics approval was granted from the Oxford Tropical Research Ethics Committee, University of Oxford and the Ethical Review Committee, University of Colombo.

On the day of the data collection, students completed a validated $\mathrm{FFQ}^{(21)}$. Demographic data were collected using the WHO Global School-based Health Survey ${ }^{(22)}$. Data from the FFQ were transcribed from the paper surveys and analysed in NutriSurvey ${ }^{(23)}$. The FFQ data were then used to generate a modified DQI-I score, which created a composite measure summarising diet quality in terms of nutritional variety, moderation, balance and adequacy. Due to limited data availability, several modifications to the original DQI-I had to be made related to the allocation of points for creating moderation and balance scores: the points for total fat and cholesterol were modified from the 
original maximum of 6 points to a new maximum of 9 points due to the lack of data on saturated fat (a component which was included in the DQI-I for a maximum of 6 points). Additional modifications were made for the 'Overall balance score'. The original DQI-I included a component related to the fatty acid ratio (PUFA:MUFA: SFA). We did not have these data, so we increased the value of the other 'Overall balance component' which was based on the macronutrient ratio. Originally, the maximum score was worth 6 points in the DQI-I, but we increased it to be worth 10 points (Table 1). The FFQ data provided a description of how many grams of food were consumed according to food group (e.g. rice, vegetables, grains, etc.). These amounts in grams were converted into daily intake of serving sizes using serving sizes from the Daily Sri Lankan Dietary Recommendations, which provide estimates of the serving sizes of rice/breads, vegetables, fruits, beans, meats and dairy products. A full description of the Sri Lankan serving sizes is provided in the online supplementary material, Supplemental Table 1.

Trained researchers weighed and measured students without their shoes and wearing only light clothing on a standard physician's beam scale (SECA 803, Hamburg, Germany) and a stadiometer (SECA 213, Hamburg, Germany). Measured height and weight were used to generate age- and sex-adjusted body weight categories (these are categories which account for the individual's age and sex when determining whether or not his/her BMI falls within a category) according to standards from the International Obesity Task Force ${ }^{(24)}$. These categories are underweight, normal weight and obese, which are extrapolated from the adult BMI cut-offs for overweight $\left(25 \mathrm{~kg} / \mathrm{m}^{2}\right)$, obesity (30 $\mathrm{kg} / \mathrm{m}^{2}$ ) and three grades of thinness (BMI of 16, 17 and $18.5 \mathrm{~kg} / \mathrm{m}^{2}$ ). It combines nationally representative survey data from the UK, USA, Netherlands, Brazil, Singapore and Hong Kong (covering the age range 2-18 years). We created a three-category body weight measure: underweight (International Obesity Task Force thinness grades 1, 2 and 3), normal weight and overweight (overweight and obese).

Based on our population's nutritional profile and the variables that were available in our data set, the DQI-I was the most suitable index for summarising diet quality among adolescents in Sri Lanka. Its suitability was based on several factors. First, the DQI-I has been validated for use internationally and it focuses on concerns related to both chronic disease and undernutrition ${ }^{(25)}$. Second, the DQI-I was compatible with the data output from the FFQ that we used in the present study ${ }^{(26)}$.

The DQI-I assesses dietary quality according to four components: its variety, adequacy, moderation and overall balance. The DQI-I is an adaptation of the original DQI, which was developed in 1999 to reflect the dietary guidelines of the time in the USA. The DQI-I was developed as a way to provide an index that would be appropriate for all countries. The index is based on worldwide and individual national dietary guidelines from the $\mathrm{WHO}^{(27)}$ and the US Department of Agriculture ${ }^{(28,29)}$. More details regarding the rationale for developing the index are documented elsewhere ${ }^{(15,30,31)}$.

The normality of continuous variables was evaluated using Shapiro-Wilk tests and normality distributions. Correlations between food or nutrient intakes and DQI-I scores were evaluated using Pearson or Spearman correlation coefficients.

We compared the means of DQI-I components by individual characteristics (sex, age, BMI category, district) using $t$ tests for binary variables or ANOVA for categorical variables. When categorical variables violated the homogeneity of variance assumption, we used the $F^{*}$ test to examine differences in DQI-I components between groups. Bonferroni corrections were used to account for the increased risk of type I errors related to multiple significance comparisons.

To examine associations between sociodemographic factors and total DQI-I score, we used multilevel modelling. Multilevel modelling was important due to the hierarchical structure of the data set (children nested within schools).

\section{Results}

Just over half of the students were female, with age ranging from 12 to 18 years (Table 2). About $43 \%$ of the sample fell into the normal weight range, while $35 \%$ were underweight. About $42 \%$ of students reported that their mothers had passed GCE/O levels (General Certificate of Education/Ordinary levels) or above, while the remaining majority reported that their mothers had an education below GCE/O level. The majority of participants identified as Sinhala (86\%), but about 9\% identified as Sri Lankan Moor and $5 \%$ as Sri Lankan Tamil.

The mean values of servings of food groups consumed according to quartile of the DQI-I score are presented in Table 3. The consumption of foods (servings/d) for all food groups (including foods high in sugar, fat and salt) increased with increasing DQI-I score. DQI-I score was positively correlated with the percentage of energy coming from protein and fat, but was negatively correlated with the percentage of energy coming from carbohydrates. DQI-I score was positively associated with mean nutrient consumption values (Table 4).

Table 5 summarises the mean values of the DQI-I component scores (variety, balance, adequacy and moderation) and the total DQI-I score according to sex, age, district, maternal education and ethnicity. After Bonferroni corrections for multiple testing, the analyses indicated that males had a significantly higher variety score compared with females, but there were no significant differences in total score. There was a significant difference in 
Table 1 Diet Quality Index-International (DQI-I) components, scores, cut-off points and baseline proportions (adapted from Kim et al. ${ }^{(25)}$ )

\begin{tabular}{|c|c|c|}
\hline Component & Possible points (pts) & Scoring \\
\hline Variety & $0-20 \mathrm{pts}$ & \\
\hline $\begin{array}{l}\text { Overall food group variety (meat/poultry/fish/eggs; dairy/ } \\
\text { beans; grain; fruit; vegetables) }\end{array}$ & $\begin{array}{c}15 \text { pts } \\
12 \\
9 \\
6 \\
3 \\
0\end{array}$ & $\begin{array}{l}\geq 1 \text { serving from each food group/d } \\
\text { Any } 1 \text { food group missing/d } \\
\text { Any } 2 \text { food groups missing/d } \\
\text { Any } 3 \text { food groups missing/d } \\
\geq 4 \text { food groups missing/d } \\
\text { None from any food groups }\end{array}$ \\
\hline $\begin{array}{l}\text { Within-group variety for protein source (meat, poultry, } \\
\text { fish, dairy, beans, eggs) }\end{array}$ & $\begin{array}{c}0-5 \text { pts } \\
5 \\
3 \\
1 \\
0\end{array}$ & $\begin{array}{l}\text { From } \geq 3 \text { different sources/d } \\
\text { From } 2 \text { different sources/d } \\
\text { From } 1 \text { source/d } \\
\text { None }\end{array}$ \\
\hline $\begin{array}{l}\text { Adequacy } \\
\text { Vegetable group }\end{array}$ & $\begin{array}{c}0-40 \text { pts } \\
0-5 \text { pts } \\
5 \\
0\end{array}$ & $\begin{array}{l}3-5 \text { servings/d } \\
0 \text { servings/d }\end{array}$ \\
\hline Fruit group & $\begin{array}{c}0-5 \text { pts } \\
5 \\
0\end{array}$ & $\begin{array}{l}2-4 \text { servings/d } \\
0 \text { servings/d }\end{array}$ \\
\hline Grain group & $\begin{array}{c}0-5 \text { pts } \\
5 \\
0\end{array}$ & $\begin{array}{l}6-11 \text { servings/d } \\
0 \text { servings/d }\end{array}$ \\
\hline Fibre & $\begin{array}{c}0-5 \text { pts } \\
5 \\
0\end{array}$ & $\begin{array}{l}\geq 30 \mathrm{~g} / \mathrm{d} \\
0 \mathrm{~g} / \mathrm{d}\end{array}$ \\
\hline Protein & $\begin{array}{c}0-5 \text { pts } \\
5 \\
0\end{array}$ & $\begin{array}{l}\geq 10 \% \text { of total energy } / \mathrm{d} \\
0 \% \text { of total energy } / \mathrm{d}\end{array}$ \\
\hline Iron & $\begin{array}{c}0-5 \text { pts } \\
5 \\
0\end{array}$ & $\begin{array}{l}\geq 100 \% \operatorname{RDA}(\mathrm{Al}) / \mathrm{d}(15 \mathrm{mg}) \\
0 \% \operatorname{RDA}(\mathrm{Al}) / \mathrm{d}\end{array}$ \\
\hline Calcium & $\begin{array}{c}0-5 \text { pts } \\
5 \\
0\end{array}$ & $\begin{array}{l}100 \% \operatorname{RDA}(\mathrm{Al}) / \mathrm{d}(1300 \mathrm{mg}) \\
0 \% \operatorname{RDA}(\mathrm{Al}) / \mathrm{d}\end{array}$ \\
\hline Vitamin C & $\begin{array}{c}0-5 \text { pts } \\
5 \\
0\end{array}$ & $\begin{array}{l}100 \% \operatorname{RDA}(\mathrm{RNI}) / \mathrm{d}(75 \mathrm{mg}) \\
0 \% \operatorname{RDA}(\mathrm{RNI}) / \mathrm{d}\end{array}$ \\
\hline $\begin{array}{c}\text { Moderation } \\
\text { Total fat }\end{array}$ & $\begin{array}{c}0-30 \text { ptst } \\
0-9 \text { pts }\end{array}$ & \\
\hline - & $\begin{array}{l}9 \\
6 \\
0\end{array}$ & $\begin{array}{l}\leq 20 \% \text { of total energy/d } \\
>20-30 \% \text { of total energy/d } \\
>30 \% \text { of total energy/d }\end{array}$ \\
\hline Cholesterol & $\begin{array}{c}0-9 \text { pts } \\
9 \\
6 \\
0\end{array}$ & $\begin{array}{l}\leq 300 \mathrm{mg} / \mathrm{d} \\
>300-400 \mathrm{mg} / \mathrm{d} \\
>400 \mathrm{mg} / \mathrm{d}\end{array}$ \\
\hline Sodium & $\begin{array}{c}0-6 \text { pts } \\
6 \\
3 \\
0\end{array}$ & $\begin{array}{l}\leq 2400 \mathrm{mg} / \mathrm{d} \\
>2400-3400 \mathrm{mg} / \mathrm{d} \\
\geq 3400 \mathrm{mg} / \mathrm{d}\end{array}$ \\
\hline Empty-calorie foods & $\begin{array}{c}0-6 \text { pts } \\
6 \\
3 \\
0\end{array}$ & $\begin{array}{l}\leq 3 \% \text { of total energy/d } \\
>3-10 \% \text { of total energy/d } \\
>10 \% \text { of total energy/d }\end{array}$ \\
\hline $\begin{array}{l}\text { Overall balance } \\
\text { Carbohydrate:protein:fat ratio (\% of total energy) }\end{array}$ & $\begin{array}{c}0-10 \mathrm{pts} \ddagger \\
10 \mathrm{pts} \\
10 \\
7 \\
3\end{array}$ & $\begin{array}{l}55-65: 10-15: 15-25 \\
52-69: 9-16: 13-27 \\
50-70: 8-17: 12-30\end{array}$ \\
\hline
\end{tabular}

Al, Adequate Intake; RNI, Reference Nutrient Intake.

†The points for total fat and cholesterol were modified from the original maximum of 6 points to a new maximum of 9 points due to the lack of data on saturated fat (a component which was included in the DQI-I for a maximum of 6 points).

¥Additional modifications were made for the 'Overall balance score'. The original DQI-I included a component related to the fatty acid ratio (PUFA:MUFA:SFA).

We did not have this data, so we increased the value of the other 'Overall balance component' which was based on the macronutrient ratio. Originally, the maximum score was worth 6 points in the DQI-I, but we increased it to be worth 10 points. 
Table 2 Demographic characteristics and BMI of the study participants: adolescents ( $n$ 1262) aged $12-18$ years living in rural Sri Lanka, 'Integrating Nutrition Promotion and Rural Development' project, August/September 2015

\begin{tabular}{|c|c|c|c|c|c|c|c|c|}
\hline & \multicolumn{2}{|c|}{ Ampara } & \multicolumn{2}{|c|}{ Moneregala } & \multicolumn{2}{|c|}{ Kurunegala } & \multicolumn{2}{|c|}{ Grand total } \\
\hline & $n$ & $\%$ & $n$ & $\%$ & $n$ & $\%$ & $n$ & $\%$ \\
\hline \multicolumn{9}{|l|}{ Sex } \\
\hline Female & 286 & 53.46 & 243 & 36.43 & 117 & 41.20 & 646 & 43.47 \\
\hline Male & 202 & 37.76 & 243 & 36.43 & 142 & $50 \cdot 00$ & 587 & 39.50 \\
\hline \multicolumn{9}{|l|}{ Age (years) } \\
\hline 12 & 13 & 2.43 & 3 & 0.45 & 2 & 0.70 & 18 & $1 \cdot 21$ \\
\hline 13 & 30 & $5 \cdot 61$ & 18 & $2 \cdot 70$ & 10 & 3.52 & 58 & 3.90 \\
\hline 14 & 35 & $6 \cdot 54$ & 39 & $5 \cdot 85$ & 22 & $7 \cdot 75$ & 96 & $6 \cdot 46$ \\
\hline 15 & 228 & $42 \cdot 62$ & 173 & 25.94 & 89 & $31 \cdot 34$ & 490 & 32.97 \\
\hline 16 & 172 & 32.15 & 245 & 36.73 & 136 & 47.89 & 553 & $37 \cdot 21$ \\
\hline 17 & 15 & $2 \cdot 80$ & 14 & $2 \cdot 10$ & 7 & 2.46 & 36 & 2.42 \\
\hline 18 & - & - & 2 & 0.30 & - & - & 2 & 0.13 \\
\hline \multicolumn{9}{|l|}{ Maternal education } \\
\hline Passed GCE/O levels or above & 164 & 33.00 & 203 & 40.93 & 160 & 59.48 & 527 & 41.76 \\
\hline Below GCE/O level & 333 & $67 \cdot 00$ & 293 & 59.07 & 109 & $40 \cdot 52$ & 735 & $58 \cdot 24$ \\
\hline \multicolumn{9}{|l|}{ Ethnicity } \\
\hline Sinhala & 336 & 67.61 & 491 & 98.99 & 259 & $96 \cdot 28$ & 1086 & 86.05 \\
\hline Sri Lankan Moor & 102 & $20 \cdot 52$ & 2 & 0.40 & 7 & $2 \cdot 60$ & 111 & 8.80 \\
\hline Indian Tamil & 2 & 0.40 & 1 & 0.20 & - & - & 3 & 0.24 \\
\hline Sri Lankan Tamil & 56 & 11.27 & 2 & 0.40 & 3 & $1 \cdot 12$ & 61 & 4.83 \\
\hline Don’t know/missing & 1 & 0.20 & - & - & - & - & 1 & 0.08 \\
\hline \multicolumn{9}{|l|}{ Age- and sex-adjusted BMI category } \\
\hline Underweight grade 3 & 46 & 8.60 & 31 & 4.65 & 23 & $8 \cdot 10$ & 100 & 6.73 \\
\hline Underweight grade 2 & 55 & $10 \cdot 28$ & 61 & $9 \cdot 15$ & 37 & 13.03 & 153 & $10 \cdot 30$ \\
\hline Underweight grade 1 & 96 & 17.94 & 118 & 17.69 & 57 & $20 \cdot 07$ & 271 & $18 \cdot 24$ \\
\hline Normal & 264 & $49 \cdot 35$ & 244 & 36.58 & 125 & 44.01 & 633 & $42 \cdot 60$ \\
\hline Overweight & 21 & 3.93 & 26 & 3.90 & 18 & 6.34 & 65 & 4.37 \\
\hline Obese & 4 & 0.75 & 6 & 0.90 & 3 & 1.06 & 13 & 0.87 \\
\hline
\end{tabular}

GCE/O level, General Certificate of Education/Ordinary level.

The percentages for each section may not add up to $100 \%$ due to observations with missing answers, which are not shown.

Table 3 Differences in consumption of daily food servings by Diet Quality Index-International (DQI-I) score categories among adolescents ( $n$ 736) aged 12-18 years living in rural Sri Lanka, based on data from the 'Integrating Nutrition Promotion and Rural Development' project, August/September 2015

\begin{tabular}{|c|c|c|c|c|c|c|c|c|c|c|c|c|c|c|}
\hline \multirow[b]{3}{*}{ Variable } & \multicolumn{12}{|c|}{ DQI-I score quartile } & \multirow[b]{3}{*}{$P$ value } & \multirow{3}{*}{$\begin{array}{l}\text { Correlation with } \\
\text { total DQI-I }\end{array}$} \\
\hline & \multicolumn{3}{|c|}{$\begin{array}{c}1 \text { st } \\
(\mathrm{DQ} / \mathrm{I}<47)\end{array}$} & \multicolumn{3}{|c|}{$\begin{array}{c}\text { 2nd } \\
\text { (DQI-I =48-56) }\end{array}$} & \multicolumn{3}{|c|}{$\begin{array}{c}3 \text { rd } \\
(\mathrm{DQI}-\mathrm{I}=57-66)\end{array}$} & \multicolumn{3}{|c|}{$\begin{array}{c}\text { 4th } \\
\text { (DQI-I =67-83) }\end{array}$} & & \\
\hline & $n$ & Mean & SD & $n$ & Mean & SD & $n$ & Mean & SD & $n$ & Mean & SD & & \\
\hline Grain (servings/d) & 277 & $6 \cdot 44$ & 2.99 & 199 & $9 \cdot 64$ & $4 \cdot 84$ & 167 & $10 \cdot 08$ & $6 \cdot 32$ & 93 & $13 \cdot 23$ & 6.52 & $<0.01$ & 0.45 \\
\hline Vegetables (servings/d) & 277 & 1.89 & $1 \cdot 19$ & 199 & $3 \cdot 20$ & 2.09 & 167 & 4.53 & 3.48 & 93 & $7 \cdot 30$ & $4 \cdot 78$ & $<0.01$ & 0.57 \\
\hline Fruit (servings/day) & 277 & 0.76 & 0.56 & 199 & 1.48 & 1.05 & 167 & $2 \cdot 35$ & 1.79 & 93 & 3.94 & $2 \cdot 19$ & $<0.01$ & 0.65 \\
\hline Dairy (servings/d) & 277 & 0.33 & 0.57 & 199 & 0.54 & 0.65 & 167 & 0.72 & 0.67 & 93 & 1.42 & 1.42 & $<0.01$ & 0.41 \\
\hline $\begin{array}{l}\text { Meat, poultry, fish and eggs } \\
\text { (servings/d) }\end{array}$ & 277 & 1.04 & 1.82 & 199 & 1.76 & $2 \cdot 12$ & 167 & 2.70 & 2.85 & 93 & $5 \cdot 25$ & 5.95 & $<0.01$ & 0.38 \\
\hline Beans (servings/d) & 277 & 0.45 & 0.36 & 199 & 0.78 & 0.53 & 167 & $1 \cdot 31$ & $1 \cdot 12$ & 93 & 2.03 & 1.44 & $<0.01$ & 0.54 \\
\hline $\begin{array}{l}\text { Foods high in fat, sugar and } \\
\text { salt (servings/d) }\end{array}$ & 277 & 3.06 & 2.84 & 199 & 5.52 & $7 \cdot 72$ & 167 & $7 \cdot 51$ & $13 \cdot 32$ & 93 & 8.89 & 8.48 & $<0.01$ & 0.27 \\
\hline
\end{tabular}

moderation score by ethnic group, with Indian Tamils having the highest moderation score.

Results from multilevel modelling indicated that after accounting for the hierarchical nature of the data set (children nested within school), total DQI-I score was significantly associated with sex, district and maternal education. There was no significant association between total score and age or ethnicity (Table 6).

DQI-I component scores and total DQI-I score by BMI status are presented in the online supplementary material,
Supplemental Table 2. There was no significant difference in DQI-I score according to BMI.

\section{Discussion}

The modified DQI-I indicated that there were significant differences between male and female secondary-school students in Sri Lanka, with males having significantly higher variety, adequacy and total DQI-I scores compared 


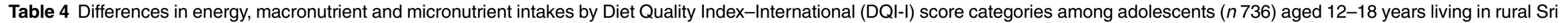
Lanka, based on data from the 'Integrating Nutrition Promotion and Rural Development' project, August/September 2015

\begin{tabular}{|c|c|c|c|c|c|c|c|c|c|c|c|c|c|c|}
\hline \multirow[b]{3}{*}{ Variable } & \multicolumn{12}{|c|}{ DQI-I score quartile } & \multirow[b]{3}{*}{$P$ value } & \multirow{3}{*}{$\begin{array}{c}\text { Correlation with } \\
\text { total DQI-I }\end{array}$} \\
\hline & \multicolumn{3}{|c|}{ 1st (DQI-I < 47) } & \multicolumn{3}{|c|}{ 2nd (DQI-I = 48-56) } & \multicolumn{3}{|c|}{ 3rd (DQI-I = 57-66) } & \multicolumn{3}{|c|}{ 4th (DQI-I = 67-83) } & & \\
\hline & $n$ & Mean & SD & $n$ & Mean & SD & $n$ & Mean & SD & $n$ & Mean & SD & & \\
\hline Energy (kJ/d) & 277 & $7680 \cdot 11$ & $2836 \cdot 21$ & 199 & 10762.59 & 3501.51 & 167 & $11466 \cdot 21$ & 3855.68 & 93 & $14410 \cdot 82$ & $5749 \cdot 23$ & $<0.01$ & 0.57 \\
\hline Energy (kcal/d) & 277 & 1835.59 & 677.87 & 199 & 2572.32 & 836.88 & 167 & 2740.49 & 921.53 & 93 & $3468 \cdot 17$ & $1374 \cdot 10$ & $<0.01$ & 0.57 \\
\hline Carbohydrates (\% energy) & 277 & 0.76 & 0.05 & 199 & 0.74 & 0.05 & 167 & 0.73 & 0.05 & 93 & 0.69 & 0.04 & $<0.01$ & -0.42 \\
\hline Protein (\% energy) & 277 & 0.09 & 0.02 & 199 & 0.09 & 0.01 & 167 & $0 \cdot 10$ & 0.01 & 93 & $0 \cdot 11$ & 0.01 & $<0.01$ & 0.29 \\
\hline Fat (\% energy) & 277 & 0.15 & 0.04 & 199 & 0.17 & 0.04 & 167 & 0.18 & 0.04 & 93 & 0.21 & 0.03 & $<0.01$ & 0.42 \\
\hline $\mathrm{Ca}(\mathrm{mg})$ & 277 & $322 \cdot 21$ & $132 \cdot 24$ & 199 & 479.28 & $182 \cdot 47$ & 167 & 576.91 & 211.38 & 93 & $882 \cdot 30$ & $416 \cdot 34$ & $<0.01$ & 0.67 \\
\hline Dietary fibre (g) & 277 & $6 \cdot 86$ & 3.95 & 199 & 12.05 & $6 \cdot 10$ & 167 & $17 \cdot 78$ & 8.49 & 93 & $29 \cdot 76$ & 11.94 & $<0.01$ & 0.74 \\
\hline Folic acid $(\mu \mathrm{g})$ & 277 & 54.72 & 24.64 & 199 & 78.94 & $32 \cdot 16$ & 167 & 85.08 & 53.22 & 93 & 118.91 & 66.67 & $<0.01$ & 0.49 \\
\hline $\mathrm{Fe}(\mathrm{mg})$ & 277 & 7.49 & 3.59 & 199 & 11.05 & 4.08 & 167 & 15.40 & $7 \cdot 19$ & 93 & 25.52 & $12 \cdot 18$ & $<0.01$ & 0.69 \\
\hline $\mathrm{Mg}(\mathrm{mg})$ & 277 & $160 \cdot 63$ & 66.69 & 199 & 248.77 & 96.69 & 167 & 302.45 & $140 \cdot 14$ & 93 & 418.09 & 149.50 & $<0.01$ & 0.67 \\
\hline$P(g)$ & 277 & 726.46 & 273.28 & 199 & 1029.02 & $306 \cdot 40$ & 167 & 1136.56 & 318.82 & 93 & 1500.39 & 560.62 & $<0.01$ & 0.65 \\
\hline $\mathrm{K}(\mathrm{mg})$ & 277 & 1199.11 & 373.58 & 199 & 1921.41 & 543.73 & 167 & 2386.03 & 867.62 & 93 & 3624.69 & 1304.64 & $<0.01$ & 0.78 \\
\hline $\mathrm{Na}(\mathrm{mg})$ & 277 & 754.56 & 421.07 & 199 & $1286 \cdot 32$ & $676 \cdot 52$ & 167 & 1713.09 & 832.52 & 93 & 2733.04 & 1211.04 & $<0.01$ & 0.67 \\
\hline Vitamin A (mg) & 277 & 343.87 & 259.46 & 199 & 625.90 & $464 \cdot 12$ & 167 & 811.94 & 722.04 & 93 & 1617.52 & 950.64 & $<0.01$ & 0.59 \\
\hline Thiamin (mg) & 277 & 1.41 & 0.54 & 199 & 1.92 & 0.63 & 167 & 2.07 & 0.58 & 93 & 2.66 & 1.01 & $<0.01$ & 0.59 \\
\hline Vitamin $B_{12}(\mathrm{mg})$ & 277 & 0.75 & 0.29 & 199 & $1 \cdot 18$ & 0.50 & 167 & 1.57 & 0.60 & 93 & $2 \cdot 19$ & 0.97 & $<0.01$ & 0.69 \\
\hline Vitamin $B_{6}(\mathrm{mg})$ & 277 & 0.47 & 0.44 & 199 & 0.78 & 0.60 & 167 & $1 \cdot 18$ & 0.73 & 93 & 2.08 & 1.31 & $<0.01$ & 0.58 \\
\hline Vitamin C (mg) & 277 & 55.50 & $50 \cdot 34$ & 199 & 117.81 & 144.07 & 167 & 164.94 & 141.29 & 93 & $286 \cdot 64$ & $217 \cdot 14$ & $<0.01$ & 0.53 \\
\hline Vitamin D $(\mu \mathrm{g})$ & 277 & 0.67 & 0.82 & 199 & 1.37 & 1.59 & 167 & 1.75 & 1.39 & 93 & 2.79 & $2 \cdot 24$ & $<0.01$ & 0.46 \\
\hline $\mathrm{Zn}(\mathrm{mg})$ & 277 & 5.44 & $2 \cdot 21$ & 199 & 7.84 & 2.31 & 167 & 8.80 & 3.02 & 93 & $12 \cdot 28$ & 4.43 & $<0.01$ & 0.67 \\
\hline
\end{tabular}




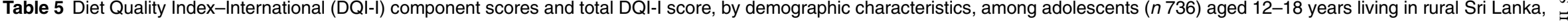
based on data from the 'Integrating Nutrition Promotion and Rural Development' project, August/September 2015

\begin{tabular}{|c|c|c|c|c|c|c|c|c|c|c|}
\hline & \multicolumn{2}{|c|}{$\begin{array}{c}\text { Variety score } \\
\text { (max: 0-20 points) }\end{array}$} & \multicolumn{2}{|c|}{$\begin{array}{c}\text { Balance score } \\
\text { (max: 0-10 points) }\end{array}$} & \multicolumn{2}{|c|}{$\begin{array}{l}\text { Adequacy score } \\
\text { (max: 0-40 points) }\end{array}$} & \multicolumn{2}{|c|}{$\begin{array}{l}\text { Moderation score } \\
\text { (max: 0-30 points) }\end{array}$} & \multicolumn{2}{|c|}{$\begin{array}{c}\text { Total score } \\
\text { (max: } 100 \text { points) }\end{array}$} \\
\hline & Mean & SD & Mean & SD & Mean & SD & Mean & SD & Mean & SD \\
\hline \multicolumn{11}{|l|}{ Sex } \\
\hline Male & $12 \cdot 88^{\star \star}$ & 0.26 & 1.17 & 2.54 & 13.04 & 8.77 & $26 \cdot 60$ & 3.17 & $53 \cdot 68$ & $12 \cdot 17$ \\
\hline Female & 11.91 & 0.26 & 1.01 & 2.43 & 11.61 & $8 \cdot 81$ & $27 \cdot 00$ & $2 \cdot 70$ & 51.53 & 11.93 \\
\hline \multicolumn{11}{|l|}{ Age (years) } \\
\hline 12 & 13.57 & $6 \cdot 16$ & 2.00 & 3.42 & 13.29 & 7.39 & 26.57 & 2.07 & 55.43 & 11.34 \\
\hline 13 & $13 \cdot 79$ & 4.89 & 1.96 & 3.06 & 14.75 & 7.96 & $26 \cdot 36$ & 3.09 & $56 \cdot 86$ & 11.65 \\
\hline 14 & $11 \cdot 13$ & 5.03 & 1.04 & 2.44 & 9.65 & $8 \cdot 32$ & $26 \cdot 75$ & 3.44 & 48.56 & 11.86 \\
\hline 15 & $12 \cdot 22$ & 5.01 & 1.02 & 2.42 & $11 \cdot 80$ & 8.54 & $26 \cdot 88$ & $2 \cdot 91$ & 51.91 & 11.54 \\
\hline 16 & $12 \cdot 74$ & 4.82 & 1.06 & 2.47 & 13.08 & $9 \cdot 24$ & $26 \cdot 73$ & 3.01 & $53 \cdot 61$ & $12 \cdot 62$ \\
\hline 17 & $13 \cdot 13$ & 4.75 & 0.81 & 1.94 & $13 \cdot 25$ & $5 \cdot 85$ & $27 \cdot 00$ & 2.45 & $54 \cdot 19$ & 6.67 \\
\hline \multicolumn{11}{|l|}{ District } \\
\hline Ampara & 12.92 & $5 \cdot 12$ & 1.30 & 2.71 & 12.93 & 9.24 & $26 \cdot 63$ & 2.64 & 53.80 & 13.06 \\
\hline Kurunegala & 12.04 & 4.75 & 0.92 & $2 \cdot 24$ & $11 \cdot 13$ & 8.83 & $26 \cdot 77$ & 3.03 & $50 \cdot 85$ & 11.69 \\
\hline Moneregala & $12 \cdot 34$ & 4.88 & 0.97 & $2 \cdot 36$ & $12 \cdot 84$ & $8 \cdot 13$ & $27 \cdot 00$ & 3.23 & $53 \cdot 15$ & $10 \cdot 97$ \\
\hline \multicolumn{11}{|l|}{ Maternal education } \\
\hline Below GCE/O level & $12 \cdot 36$ & 4.99 & 1.07 & 2.47 & $12 \cdot 01$ & $8 \cdot 61$ & 27.06 & 2.74 & $52 \cdot 50$ & $12 \cdot 10$ \\
\hline Passed GCE/O levels or above & 12.57 & 4.90 & $1 \cdot 10$ & 2.48 & $12 \cdot 72$ & 8.97 & $26 \cdot 54$ & 3.13 & 52.93 & 12.07 \\
\hline \multicolumn{11}{|l|}{ Ethnicity } \\
\hline Sinhala & 12.45 & 4.91 & 1.08 & 2.49 & $12 \cdot 41$ & $8 \cdot 81$ & $26 \cdot 77^{\star \star}$ & 3.01 & $52 \cdot 71$ & 12.04 \\
\hline Sri Lankan Tamil & 12.05 & 5.58 & 1.76 & $2 \cdot 81$ & 11.24 & $9 \cdot 86$ & 26.86 & 3.21 & 51.90 & $12 \cdot 88$ \\
\hline Indian Tamil & $12 \cdot 00$ & 8.49 & 1.50 & $2 \cdot 12$ & $15 \cdot 50$ & 14.85 & 28.50 & $2 \cdot 12$ & 57.50 & $23 \cdot 33$ \\
\hline Sri Lankan Moor & $13 \cdot 21$ & 5.25 & 0.59 & 1.86 & 12.55 & 7.94 & $27 \cdot 10$ & 1.70 & 53.45 & 12.07 \\
\hline
\end{tabular}

GCE/O level, General Certificate of Education/Ordinary level.

${ }^{* \star} P<0.01$. 
Table 6 Sociodemographic factors associated with Diet Quality Index-International total score among $(n 736)$ aged $12-18$ years living in rural Sri Lanka, based on multilevel modelling of data from the 'Integrating Nutrition Promotion and Rural Development' project, August/September 2015

\begin{tabular}{|c|c|c|}
\hline & $\beta$ & $95 \% \mathrm{Cl}$ \\
\hline Constant & $52 \cdot 17$ & $41 \cdot 69,62 \cdot 64$ \\
\hline \multicolumn{3}{|l|}{ Sex } \\
\hline Male & \multicolumn{2}{|c|}{ Reference category } \\
\hline Female & $-2 \cdot 49^{\star}$ & $-4.24,-0.75$ \\
\hline \multicolumn{3}{|l|}{ Age (years) } \\
\hline 12 & \multicolumn{2}{|c|}{ Reference category } \\
\hline 13 & 3.76 & $-7 \cdot 19,14.71$ \\
\hline 14 & -0.70 & $-11.37,9.96$ \\
\hline 15 & 1.96 & $-8 \cdot 34,12 \cdot 26$ \\
\hline 16 & 4.08 & $-6 \cdot 29,14 \cdot 44$ \\
\hline 17 & 3.41 & $-8 \cdot 32,15 \cdot 14$ \\
\hline \multicolumn{3}{|l|}{ District } \\
\hline Ampara & \multicolumn{2}{|c|}{ Reference category } \\
\hline Kurunegala & $-5 \cdot 21^{*}$ & $-9.53,-0.89$ \\
\hline Moneregala & $-2 \cdot 48^{*}$ & $-6.35,1.39$ \\
\hline \multicolumn{3}{|l|}{ Maternal education } \\
\hline Below GCE/O level & \multicolumn{2}{|c|}{ Reference category } \\
\hline Passed GCE/O levels or above & $1.87^{*}$ & $0.06,3.68$ \\
\hline \multicolumn{3}{|l|}{ Ethnicity } \\
\hline Sinhala & \multicolumn{2}{|c|}{ Reference category } \\
\hline Sri Lankan Tamil & $-1 \cdot 80$ & $-8 \cdot 38,4.78$ \\
\hline Indian Tamil & 0.98 & $-14 \cdot 81,16 \cdot 78$ \\
\hline Sri Lankan Moor & -0.38 & $-5 \cdot 65,4.88$ \\
\hline
\end{tabular}

GCE/O level, General Certificate of Education/Ordinary level.

${ }^{*} P<0.05$.

with females. Multilevel analyses indicated that sex, district and maternal education were all significantly associated with diet quality.

Results from the present study indicate suboptimal dietary intakes: carbohydrates provided over $70 \%$ of energy, protein provided about $10 \%$ and the remaining energy came from fat. These findings are compatible with data collected from adults ${ }^{(32)}$. Higher DQI-I score corresponded to a lower proportion of carbohydrates and higher proportions of protein and fats in the diet. Similarly, research among Sri Lankan adults showed that higher BMI and obesity were associated with higher dietary diversity and variety scores ${ }^{(33)}$. We observed that girls had a lower energy intake than boys, which corroborates findings from a study with Sri Lankan adults indicating that women had lower energy intake than men, mainly due to smaller portion sizes ${ }^{(32)}$.

Differences in dietary nutritional quality according to sex have been observed before. Qualitative data collected prior to the current project suggested concern with maintaining a low body weight among subsets of this population, which may contribute to some of the observed differences in diet quality among males and females ${ }^{(34)}$.

We found that maternal education, too, was significantly associated with diet quality score. This is consistent with other findings, including that maternal education is important in reducing the risk of anaemia and Fe deficiency and increasing children's consumption of animal-source foods ${ }^{(35)}$ and that dietary diversity is associated with child nutritional status ${ }^{(36)}$. Research also suggests that maternal education is one of the most important factors in explaining differentials in child health outcomes ${ }^{(37)}$.

The present study had several strengths and weaknesses. To the best of our knowledge, while work has been done to evaluate diets using the DQI-I in a wide range of countries, including China $^{(25)}$, Spain ${ }^{(38)}$ and Canada ${ }^{(39)}$, the DQI-I has never been applied to data from Sri Lanka. This is important because it allows for comparison between populations. DQI-I allocates higher scores to diets that are low in fat and high in carbohydrates. However, recent evidence suggests that highcarbohydrate diets (rather than diets high in fats) are associated with increased risk of $\mathrm{CVD}^{(40)}$.

Limitations of the study include the fact that the FFQ that we used was validated for adults, but it was not validated for use with adolescent populations ${ }^{(26)}$. We observed wide variation in dietary outcomes between students, which was reflected by high standard deviations. For example, twenty-three students reported to consume more than 20 servings of vegetables. Relying on self-reported data, it was not possible to verify the accuracy of these reports. Additional work is needed to identify the best methods for assessing dietary intake among adolescents.

Additionally, the present study was limited by the lack of data on saturated and trans fats in the diet, which meant that the scoring may have been weakened because it could not differentiate between healthy and unhealthy fats. An additional limitation is related to the scoring methods used in the DQI-I, which employed binary measures for categories such as adequacy, where participants could get 0 points for inadequate consumption or 5 points for adequate consumption. An improved score might give participants, for example, 2 or 3 points if they almost meet recommendations.

\section{Conclusion}

In conclusion, our findings indicate that DQI-I score among adolescents in Sri Lanka is positively associated with micronutrient consumption and negatively associated with the percentage of total energy coming from carbohydrates. This finding is important, especially in a context where individuals may face food insecurity and, given the lack of affordable, nutrient-dense foods such as fruits, vegetables, dairy and other proteins, may resort to energydense micronutrient-poor sources of food.

Our findings indicate important differences in DQI-I score according to sex and maternal education. Further work is needed to explore why diet quality is poor among females; qualitative work by Townsend et al. indicates that, as in many parts of the world, adolescent girls in rural Sri Lanka are preoccupied with maintaining a low body weight, and this may come at the expense of getting adequate nutrition. It is also possible that females have a lower DQI-I because they eat less, owing to lower nutritional needs than boys. 
The finding that low maternal education may have an adverse effect on dietary quality suggests that interventions must target low socio-economic groups, and communitybased methods which promote education to both adolescents and their families may be an important intervention. The association between district and dietary quality suggests that a 'one size fits all' approach may not be optimal, and interventions may need to be targeted for specific districts.

To improve diet quality, interventions must increase the availability and affordability of healthier foods through programmes that provide food at school or those that target economic development. Interventions will require involvement from many stakeholders from within the government, non-governmental organisations and other community groups.

\section{Acknowledgements}

Financial support: This project was funded by the Integrating Nutrition Promotion and Rural Development (INPARD) project, which was supported by the South Asian Food and Nutrition Security Initiative (SAFANSI) Trust Fund of the World Bank. J.W. is supported by a DPhil scholarship from the Nuffield Department of Population Health, University of Oxford. K.W. and N.T. are supported by a grant from the British Heart Foundation (006/P\&C/CORE/2013/OXFSTATS). The authors thank INPARD partners, data collectors and Reawakening Project Staff, and acknowledge school principals who participated in the study and education authorities who granted permission for school staff to take part in this project. The funders had no role in the design, analysis or writing of this article. Conflict of interest: None. Authorship: K.W., S.M., P.K., N.T., M.R., R.J. and J.W. conceived of and designed the study and acquired the data. J.W. analysed the data and drafted the manuscript. K.W., S.M., P.K., N.T., M.R. and R.J. revised the manuscript critically for intellectual content. K.W., S.M., P.K., N.T., M.R., R.J. and J.W. approved the version of the manuscript to be published. Ethics of human subject participation: This study was conducted according to the guidelines laid down in the Declaration of Helsinki and all procedures involving human subjects were approved by the Universities of Oxford and Colombo. Written informed consent was obtained from all subjects.

\section{Supplementary material}

To view supplementary material for this article, please visit https://doi.org/10.1017/S1368980019000430

\section{References}

1. Story M \& Alton I (1996) Adolescent nutrition: current trends and critical issues. Top Clin Nutr 11, 56-69.

2. World Health Organization (2012) Sri Lanka: Main public health issues and concerns. https://www.who.int/hac/ donorinfo/lka/en/index1.html (accessed February 2019).
3. De Silva WI (2012) Demographic dividend and economic growth in Sri Lanka. Asian Popul Stud 8, 249-250.

4. Wickramasinghe $\mathrm{V}$, Lamabadusuriya $\mathrm{S}$, Atapattu $\mathrm{N}$ et al. (2004) Nutritional status of schoolchildren in an urban area of Sri Lanka. Ceylon Med J 49, 114-118.

5. Katulanda P, Jayawardena M, Sheriff M et al. (2010) Prevalence of overweight and obesity in Sri Lankan adults. Obes Rev 11, 751-756.

6. Katulanda P, Ranasinghe P, Jayawardana R et al. (2012) Metabolic syndrome among Sri Lankan adults: prevalence, patterns and correlates. Diabetol Metab Syndr 4, 24.

7. Katulanda P, Constantine G, Mahesh J et al. (2008) Prevalence and projections of diabetes and pre-diabetes in adults in Sri Lanka - Sri Lanka Diabetes, Cardiovascular Study (SLDCS). Diabet Med 25, 1062-1069.

8. Hettiarachchi $\mathrm{M}$, Liyanage $\mathrm{C}$, Wickremasinghe $\mathrm{R}$ et al. (2006) Prevalence and severity of micronutrient deficiency: a cross-sectional study among adolescents in Sri Lanka. Asia Pac J Clin Nutr 15, 56-63.

9. Rathnayake KM, Wimalathunga M, Weech M et al. (2015) High prevalence of undernutrition and low dietary diversity in institutionalised elderly living in Sri Lanka. Public Health Nutr 18, 2874-2880.

10. Ruel MT, Harris J \& Cunningham K (2013) Diet quality in developing countries. In Diet Quality: An Evidence-based Approach, vol. 2, pp. 239-261 [VR Preedy, L-A Hunter and V Patel, editors]. New York: Springer.

11. Jayawardena R, Byrne NM, Soares MJ et al. (2013) Food consumption of Sri Lankan adults: an appraisal of serving characteristics. Public Health Nutr 16, 653-658.

12. Fransen HP \& Ocké MC (2008) Indices of diet quality. Curr Opin Clin Nutr Metab Care 11, 559-565.

13. Hann CS, Rock CL, King I et al. (2001) Validation of the Healthy Eating Index with use of plasma biomarkers in a clinical sample of women. Am J Clin Nutr 74, 479-486.

14. Kant AK, Schatzkin A \& Ziegler RG (1995) Dietary diversity and subsequent cause-specific mortality in the NHANES I epidemiologic follow-up study. J Am Coll Nutr 14, 233-238.

15. Kennedy E, Ohls J, Carlson S et al. (1995) The Healthy Eating Index: design and applications. J Am Diet Assoc 95, 1103-1108.

16. Vandevijvere S, Monteiro C, Krebs-Smith S et al. (2013) Monitoring and benchmarking population diet quality globally: a step-wise approach. Obes Rev 14, 135-149.

17. Waijers PM, Feskens EJ \& Ocké MC (2007) A critical review of predefined diet quality scores. BrJ Nutr 97, 219-231.

18. Arvaniti F \& Panagiotakos DB (2008) Healthy indexes in public health practice and research: a review. Crit Rev Food Sci Nutr 48, 317-327.

19. Marshall S, Burrows T \& Collins C (2014) Systematic review of diet quality indices and their associations with healthrelated outcomes in children and adolescents. J Hum Nutr Diet 27, 577-598.

20. World Health Organization (2013) Informed Consent Form Templates. http://www.who.int/rpc/research_ethics/informed_ consent/en (accessed February 2019).

21. Jayawardena R, Swaminathan S, Byrne NM et al. (2012) Development of a food frequency questionnaire for Sri Lankan adults. Nutr J 11, 63.

22. World Health Organization (2015) Global school-based student health survey (GSHS). https://www.who.int/ncds/ surveillance/gshs/en/ (accessed February 2019).

23. Nutrition Surveys and Calculations (2010) Homepage. http://www.nutrisurvey.de/ (accessed February 2019).

24. Cole TJ \& Lobstein T (2012) Extended international (IOTF) body mass index cut-offs for thinness, overweight and obesity. Pediatr Obes 7, 284-294.

25. Kim S, Haines PS, Siega-Riz AM et al. (2003) The Diet Quality Index-International (DQI-I) provides an effective tool for cross-national comparison of diet quality as illustrated by China and the United States. J Nutr 133, 3476-3484. 
26. Jayawardena R, Byrne NM, Soares M et al. (2016) Validity of a food frequency questionnaire to assess nutritional intake among Sri Lankan adults. Springerplus 5, issue 1, 162.

27. World Health Organization (1998) Preparation and Use of Food-Based Dietary Guidelines. Report of a Joint FAO/WHO Consultation. WHO Technical Report Series no. 880. Geneva: WHO.

28. US Department of Agriculture (2016) Dietary Guidelines from Around the World. https://www.nal.usda.gov/fnic/dietaryguidelines-around-world (accessed December 2016).

29. US Department of Agriculture (1992) The Food Guide Pyramid. Home and Garden Bulletin no. 252. Washington, DC: US Department of Agriculture, Center for Nutrition Policy and Promotion.

30. Patterson RE, Haines PS \& Popkin BM (1994) Diet Quality Index: capturing a multidimensional behavior. $J$ Am Diet Assoc 94, 57-64.

31. Haines PS, Siega-Riz AM \& Popkin BM (1999) The Diet Quality Index revised: a measurement instrument for populations. J Am Diet Assoc 99, 697-704.

32. Jayawardena R, Thennakoon S, Byrne N et al. (2014) Energy and nutrient intakes among Sri Lankan adults. Int Arch Med 7, 34 .

33. Jayawardena R, Byrne NM, Soares MJ et al. (2013) High dietary diversity is associated with obesity in Sri Lankan adults: an evaluation of three dietary scores. BMC Public Health 13, 314.

34. Townsend N, Williams J, Wickramasinghe K et al. (2015) Barriers to healthy dietary choice amongst students in Sri Lanka as perceived by school principals and staff. Health Promot Int 32, 91-101.

35. Choi H-J, Lee H-J, Jang HB et al. (2011) Effects of maternal education on diet, anemia, and iron deficiency in Korean school-aged children. BMC Public Health 11, 870.

36. Arimond M \& Ruel MT (2004) Dietary diversity is associated with child nutritional status: evidence from 11 demographic and health surveys. J Nutr 134, 2579-2585.

37. Frost MB, Forste R \& Haas DW (2005) Maternal education and child nutritional status in Bolivia: finding the links. $S O C$ Sci Med 60, 395-407.

38. Tur JA, Romaguera D \& Pons A (2005) The Diet Quality Index-International (DQI-I): is it a useful tool to evaluate the quality of the Mediterranean diet? Br J Nutr $\mathbf{9 3}$, 369-376.

39. Florence MD, Asbridge M \& Veugelers PJ (2008) Diet quality and academic performance. $J$ Sch Health 78, 209-215.

40. Dehghan M, Mente A, Zhang X et al. (2017) Associations of fats and carbohydrate intake with cardiovascular disease and mortality in 18 countries from five continents (PURE): a prospective cohort study. Lancet 390, 2050-2062. 